\title{
AN INTERFACE REGION IMAGING SPECTROGRAPH FIRST VIEW ON SOLAR SPICULES
}

\author{
T. M. D. Pereira ${ }^{1}$, B. De Pontieu ${ }^{1,2}$, M. Carlsson ${ }^{1}$, V. Hansteen ${ }^{1}$, T. D. Tarbell ${ }^{2}$, J. Lemen ${ }^{2}$, A. Title ${ }^{2}$, P. Boerner ${ }^{2}$, \\ N. Hurlburt ${ }^{2}$, J. P. Wülser ${ }^{2}$, J. Martínez-Sykora ${ }^{2,3}$, L. Kleint ${ }^{2,3,4}$, L. Golub ${ }^{5}$, S. McKillop ${ }^{5}$, K. K. Reeves ${ }^{5}$, S. SaAR ${ }^{5}$,

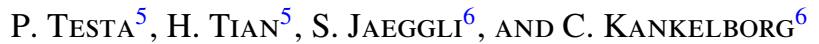 \\ ${ }^{1}$ Institute of Theoretical Astrophysics, University of Oslo, P.O. Box 1029 Blindern, NO-0315 Oslo, Norway; tiago.pereira@ astro.uio.no \\ ${ }^{2}$ Lockheed Martin Solar and Astrophysics Laboratory, 3251 Hanover Street, Org. A021S, Bldg. 252, Palo Alto, CA 94304, USA \\ ${ }^{3}$ Bay Area Environmental Research Institute, 596 1st Street West, Sonoma, CA 95476, USA \\ ${ }^{4}$ NASA Ames Research Center, Moffett Field, CA 94035, USA \\ ${ }^{5}$ Harvard-Smithsonian Center for Astrophysics, 60 Garden Street, Cambridge, MA 02138, USA \\ ${ }^{6}$ Department of Physics, Montana State University, P.O. Box 173840, Bozeman, MT 59717, USA \\ Received 2014 June 23; accepted 2014 July 23; published 2014 August 19
}

\begin{abstract}
Solar spicules have eluded modelers and observers for decades. Since the discovery of the more energetic type II, spicules have become a heated topic but their contribution to the energy balance of the low solar atmosphere remains unknown. Here we give a first glimpse of what quiet-Sun spicules look like when observed with NASA's recently launched Interface Region Imaging Spectrograph (IRIS). Using IRIS spectra and filtergrams that sample the chromosphere and transition region, we compare the properties and evolution of spicules as observed in a coordinated campaign with Hinode and the Atmospheric Imaging Assembly. Our IRIS observations allow us to follow the thermal evolution of type II spicules and finally confirm that the fading of $\mathrm{Ca}$ II $\mathrm{H}$ spicules appears to be caused by rapid heating to higher temperatures. The IRIS spicules do not fade but continue evolving, reaching higher and falling back down after 500-800 s. Ca II H type II spicules are thus the initial stages of violent and hotter events that mostly remain invisible in Ca II $\mathrm{H}$ filtergrams. These events have very different properties from type I spicules, which show lower velocities and no fading from chromospheric passbands. The IRIS spectra of spicules show the same signature as their proposed disk counterparts, reinforcing earlier work. Spectroheliograms from spectral rasters also confirm that quiet-Sun spicules originate in bushes from the magnetic network. Our results suggest that type II spicules are indeed the site of vigorous heating (to at least transition region temperatures) along extensive parts of the upward moving spicular plasma.
\end{abstract}

Key words: Sun: atmosphere - Sun: chromosphere - Sun: transition region

Online-only material: animations, color figures

\section{INTRODUCTION}

A better understanding of solar spicules is essential to unlock the mysteries of the chromosphere and low solar atmosphere. These dynamic jet-like features dominate the solar limb and since their first reports they have been challenging to explain, as attested in various reviews (Beckers 1968; Sterling 2000; Tsiropoula et al. 2012). Over the last several decades, spicules have been observed in a variety of filters and instruments (e.g., Dere et al. 1989; Wilhelm 2000; Zachariadis \& Gontikakis 2002; O'Shea et al. 2005), but the contribution of the Hinode mission (Kosugi et al. 2007) has been nothing short of revolutionary. From high-quality time series of spicules in the $\mathrm{Ca}$ II $\mathrm{H}$ band of the Solar Optical Telescope (SOT; Tsuneta et al. 2008; Suematsu et al. 2008), it emerged that there are at least two types of spicules (De Pontieu et al. 2007). Type I spicules have a slower rise and fall (15-40 $\mathrm{km} \mathrm{s}^{-1}$ ) and long lifetimes (3-10 minutes), with a parabolic motion in space-time diagrams, while type II spicules have a faster upward rise $\left(30-110 \mathrm{~km} \mathrm{~s}^{-1}\right)$ followed by a fading from the Ca II $\mathrm{H}$ passband after 50-150 s; they are not observed to fall back down (De Pontieu et al. 2007; Pereira et al. 2012, hereafter Paper I). Type II spicules are the most abundant (seen in quiet Sun and coronal holes), while type I spicules appear mostly in and around active regions.

The disappearance of type II spicules from the $\mathrm{Ca}$ II $\mathrm{H}$ passband has fueled speculation that these may be heated to higher temperatures (De Pontieu et al. 2009; McIntosh \& De Pontieu 2009; McIntosh et al. 2010; Tian et al. 2011), possibly even to transition region (TR) or coronal temperatures, as suggested by De Pontieu et al. (2011). However, establishing the thermal evolution of spicules to the higher chromosphere and TR has been difficult because the spatial and temporal resolution of previous instruments is just barely enough to resolve their rapid motions (see also Pereira et al. 2013a). The recently launched IRIS mission (De Pontieu et al. 2014) is aimed precisely at observing the dynamic interface between the chromosphere and corona, therefore making it ideal for the study of spicules. The aim of this work is to provide an overview of the quiet-Sun spicule dynamics and thermal evolution as observed with IRIS, comparing them with the $\mathrm{Ca}$ II $\mathrm{H}$ spicules from Hinode/SOT and their higher temperature counterparts from the Atmospheric Imaging Assembly (AIA; Lemen et al. 2012).

\section{OBSERVATIONS AND ANALYSIS}

We make use of a series of coordinated quiet-Sun observations obtained with IRIS, Hinode/SOT, and AIA, taken on 2014 February 21. From IRIS we use the chromospheric and TR slitjaw filtergrams at $279.6 \mathrm{~nm}$ (dominated by $\mathrm{Mg}$ II, at $\approx 10 \mathrm{kK}$ ) and $140.0 \mathrm{~nm}$ (dominated by Si IV, at $\approx 80 \mathrm{kK}$ under ionization equilibrium conditions), taken with a $19 \mathrm{~s}$ cadence. From SOT, we use the $396.85 \mathrm{~nm}$ filtergrams (dominated by $\mathrm{Ca}$ II $\mathrm{H}$, at $\approx 9 \mathrm{kK}$ ) from the broadband filter imager, taken with a $4.8 \mathrm{~s}$ cadence. From AIA, we use the $30.4 \mathrm{~nm}$ filtergrams (dominated by He II, at $\approx 100 \mathrm{kK}$ ), taken with a $12 \mathrm{~s}$ cadence. The target of the observations was the quiet Sun at the south pole (no coronal 
hole was visible), and the time series duration was 91 minutes. The IRIS field of view was centered at $(x, y)=\left(6.7,-965^{\prime \prime} .5\right)$.

We made use of IRIS calibrated level 2 data (for details on the reduction, see De Pontieu et al. 2014). The SOT images were reduced using the same procedure as in Paper I. For AIA, we used calibrated level 1.5 images (see Lemen et al. 2012). In all IRIS and SOT filtergrams shown, we applied a radial density filter (see De Pontieu et al. 2007) to enhance the visibility of spicules. The IRIS, SOT, and AIA spatial resolutions are approximately $00^{\prime} .33,0^{\prime \prime} .2$, and $1^{\prime \prime} .5$, respectively. The SOT and AIA images were aligned and interpolated to the IRIS pixel size of 0 .' 166 pixel $^{-1}$.

Additionally, we make use of a very large, dense raster observed with IRIS at the solar north pole on 2013 October 9 at 13:10 UT. This raster has 400 positions observed with a spatial spacing of 0.35 and a cadence of $9 \mathrm{~s}$. The raster covered about $140^{\prime \prime} \times 175^{\prime \prime}$ and took $1 \mathrm{hr}$ to complete. The spacecraft orbital velocity and spectrograph thermal drifts were compensated by fitting the position of the Ni I $279.947 \mathrm{~nm}$ line and subtracting its long-term trend from the signal.

\section{RESULTS}

\subsection{Morphology}

In Figure 1 we show a comparison between the IRIS, SOT, and AIA filtergrams. The $\mathrm{Mg}$ II $\mathrm{k}$ spicules are seen as a natural upward extension of the $\mathrm{Ca}$ II $\mathrm{H}$ spicules. They are consistently taller and in several cases twice as tall. The Si IV spicules show a continuation of the $\mathrm{Mg}$ II spicules and expand even farther. From the lower panel of Figure 1 one can see that the Si IV spicules extend just above the Mg II spicules virtually everywhere. Even accounting for the different spatial resolution, the IRIS spicules are broader and more nebulous than in SOT, where individual spicule "strands" are seen more clearly.

Only the longest spicules are seen in the He II 30.4 filter, because it is opaque in higher layers that obscure the shorter spicules. He II spicules extend just above the Si IV spicules and their shape is blurrier, partly because of the lower spatial resolution, and presumably in part because of the more complicated radiative transfer.

Many of the Si IV spicules have a much darker bottom third or half, with the top part of the spicule noticeably brighter. This is not generally seen in Mg II or Ca II, and indicates that the bottom part of several spicules may not be hot enough to have significant $\mathrm{Si}$ IV emission. This is clearly shown in the top panels of Figure 1 (and in the space-time diagrams of the same spicule A in Figure 3 ). Here, the Si IV emission occurs primarily in the top half of the spicule, a situation that is relatively common.

\subsection{Time Evolution}

The spicules observed were mostly of type II, showing a fast rise $\left(>50 \mathrm{~km} \mathrm{~s}^{-1}\right)$ and then fading from the Ca II $\mathrm{H}$ filtergrams. In the IRIS filtergrams, they have a similar upward velocity but continue to rise after they fade in Ca II, reaching heights up to $20 \mathrm{Mm}$ above the limb and often falling down later in a parabolic motion. In several cases, this rise and fall is also seen in the He II $30.4 \mathrm{~nm}$ filtergrams. In Figure 2 we show one such event where a spicule fades in Ca II but continues to evolve in the IRIS and AIA filters. This suggests that such spicules are being heated out of the Ca II passband, and continue evolving reaching higher layers and temperatures. This extended evolution leads to longer spicule lifetimes in the IRIS images, typically 500-800 s.
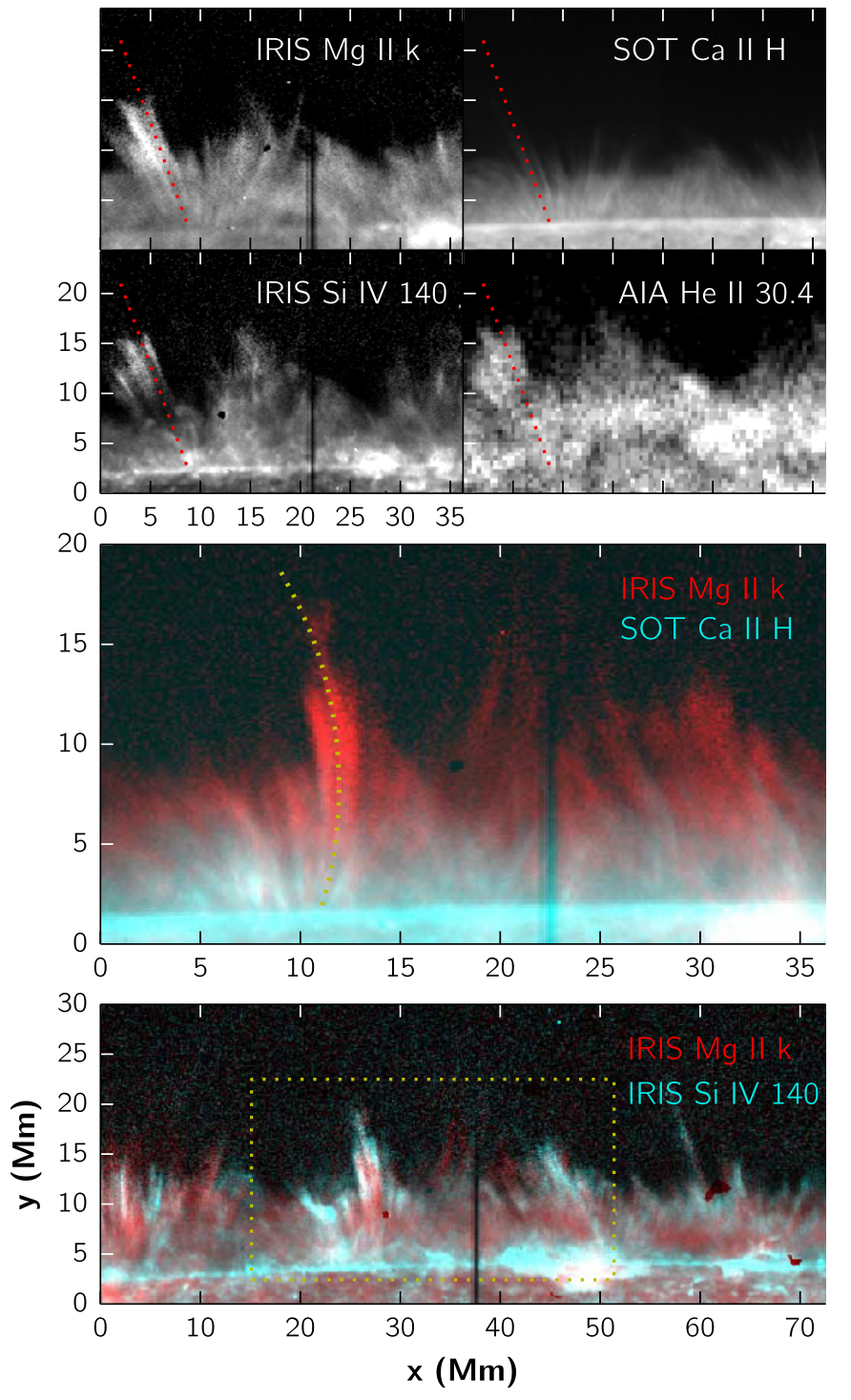

Figure 1. Quiet-Sun spicule images from 2014 February 21, for two of IRIS slit-jaw filtergrams, SOT Ca II H filtergrams, and the AIA He II $30.4 \mathrm{~nm}$ channel. Top four panels: observations at 11:42:11 UT; the dotted line is the region used to build the space-time diagram for spicule A in Figure 3. Middle panel: color composite of observations at 12:19:31 UT, where the red channel corresponds to the IRIS Mg II k filtergram and the green and blue channels (cyan color) to the SOT Ca II H filtergram. The dotted line is the spicule axis (at this instant) used to build the diagram for spicule B in Figure 3. Bottom panel: composite image using the IRIS Mg II k and Si IV filtergrams, for the same instant as the middle panel and a larger field. The dotted rectangle denotes the field-of-view of the middle panel.

(Animations and color version of this figure are available in the online journal.)

The spicule in Figure 2 is in fact a rare case of a type II spicule that is observed to fall back down in $\mathrm{Ca}$ II much later after it fades. At $t \approx 512.7 \mathrm{~s}$ in the intensity and in the space-time diagram, it is possible to discern a faint trail from a descending spicule, roughly at the same time when the spicule is observed to fall back down by IRIS. With only the $\mathrm{Ca}$ II images it would be difficult to associate this falling material with the original spicule, but taking into account the IRIS images it becomes clear that this is most likely falling material that has cooled down and therefore is visible again in Ca II. In Figure 2 there are further examples of the thermal evolution of spicules. In the first frames the later stages of a different spicule are seen on the left side of the IRIS and AIA panels, which had already faded 

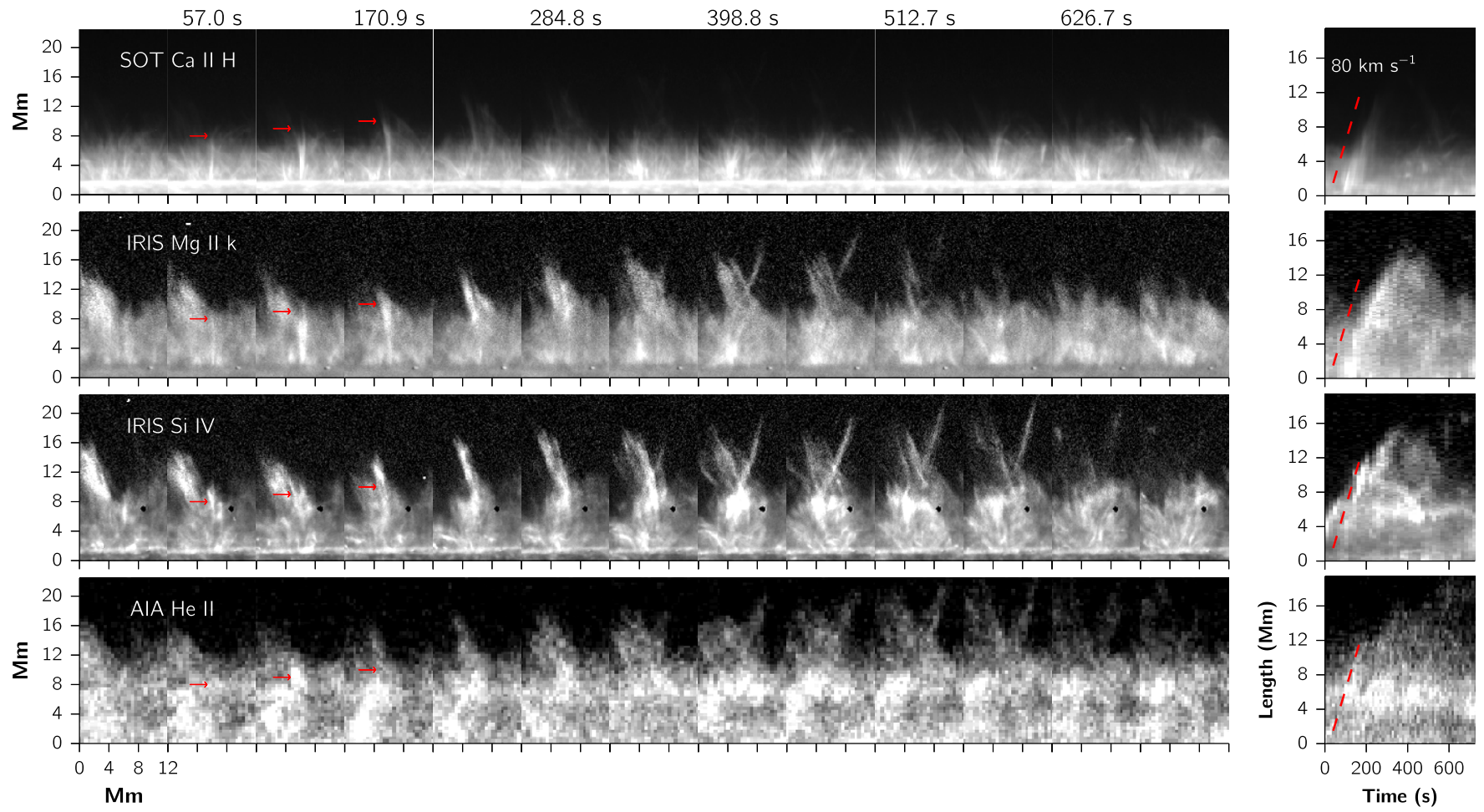

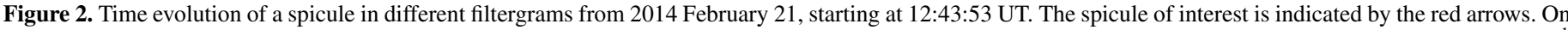

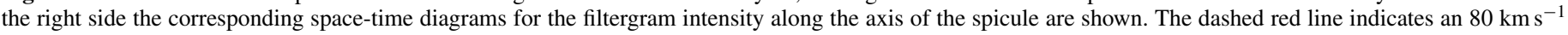
trajectory.

(A color version of this figure is available in the online journal.)

from the Ca II image. At around $t \approx 350 \mathrm{~s}$, a different spicule rises diagonally, toward the upper right corner. This spicule is hardly visible in Ca II but prominent in the other filters; it is presumably undergoing more vigorous heating.

In Figure 3 we show space-time diagrams for two other spicules, illustrating the differences in temporal evolution for different filters. Again, the spicules show a clear parabolic trajectory and similar lifetimes in all filters but $\mathrm{Ca}$ II $\mathrm{H}$. Spicule A shows a common evolution where Si IV is much brighter at the top of the spicule, and toward the later stages of its life. A close look shows that a very faint outline of the near-parabolic trajectory of spicule A is also traced in Ca II. Spicule B also shows more Si IV emission near its top. In some cases the Si IV space-time diagrams look almost like half a parabola, brightening up near the later life of the spicule and coinciding with the instant where it fades in Ca II.

\subsection{Spectral Rasters}

In Figure 4 we show an overview of a dense raster. Positive velocities are defined as toward the observer. The $\mathrm{Mg}$ II $\mathrm{h}$ and $\mathrm{k}$ lines have the advantages of a wide formation range (Leenaarts et al. 2013a, 2013b; Pereira et al. 2013b) and a core that is much brighter than the wings. From Figure 4 it is clear how the disk counterparts of spicules arise naturally in bushes when observed in the wings of the $\mathrm{k}$ line (see also Beckers 1968). As one observes in wavelengths closer to the line core there are more spicules per bush. At the line core there is more opacity from the chromospheric canopy and the spicule footpoints are no longer visible. Dopplergrams (constructed by subtracting red and blue wing images at the same wavelength offset) provide a view of the spicule line-of-sight velocities (caused by the three kinds of spicule motion: upflow/downflow, transverse, and torsional; see Sekse et al. 2013) and how spicule numbers increase when closer to the line core.

In the middle right and bottom right panels of Figure 4, we show the mean properties of the $\mathrm{k}$ line as function of the distance to the limb. The equivalent width (EW), integrated from 100 to $-100 \mathrm{~km} \mathrm{~s}^{-1}$ from the line core, decreases by half of its limb value at about $4.5 \mathrm{Mm}$ above the limb. The $\mathrm{Mg}$ II lines get broader from the disk to the limb; the FWHM increases sharply just after the limb, peaking at $\approx 1.8 \mathrm{Mm}$ above the limb and then falling steeply. In the spatially averaged spectra, the Mg II lines are strong even at considerable distances from the limb (here defined as zero at $280.9 \mathrm{~nm}$ ). The $\mathrm{k}$ line shows a double peak until about $6 \mathrm{Mm}$ above the limb, and a single-peaked emission profile is still seen beyond $12 \mathrm{Mm}$ above the limb. In the insets in the middle right panels of the figure, we show sample spectra from adjacent spicules, both on disk and off-limb. These spectra show the same extended wings as the rapid blue-shifted events (RBEs) and rapid red-shifted events (RREs) observed on disk in other lines (Langangen et al. 2008; Rouppe van der Voort et al. 2009; Sekse et al. 2013), confirming that off-limb spicules and disk RBEs/RREs have the same spectral signature.

In the bottom left panel of Figure 4, we show a C II $133 \mathrm{~nm}$ slit-jaw image taken at a particular instant of the raster. Because of the long exposure of the raster, not all the structures seen in the slit-jaw are co-temporal with the features in the raster. Nevertheless, it is still a useful comparison. The C II image shows the limb spicules and also the brighter of their disk counterparts. The fainter disk features are not resolved in the C II image, but instead appear as cloudy areas (see also Tian et al. 2014). Almost all of these cloudy features appear in the 


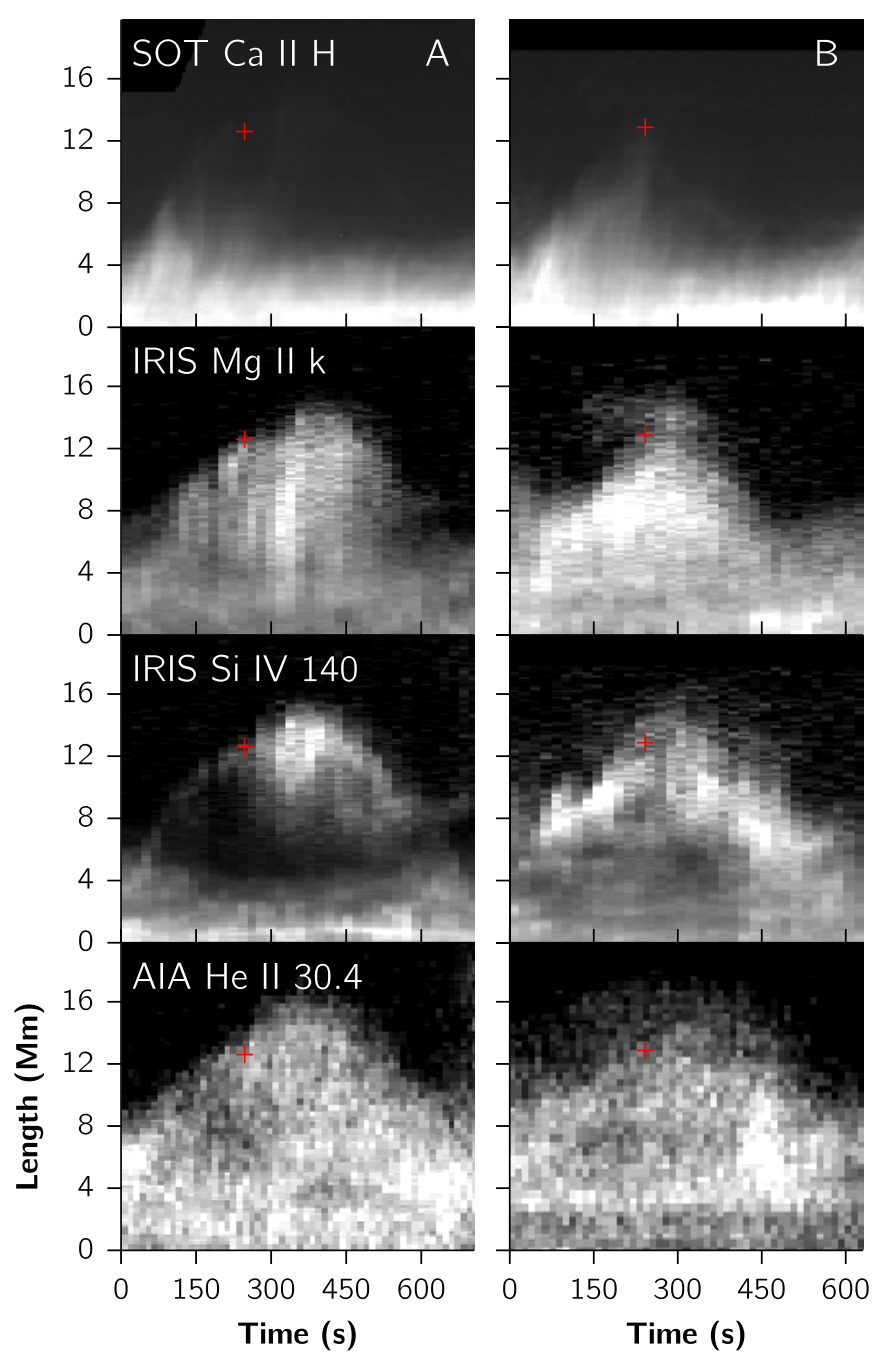

Figure 3. Space-time diagrams for two spicules (A and B, left and right columns, respectively). Diagrams show the filtergram intensity along the axis of the spicules (following the transverse motion). The red crosses show the approximate maximum length of the spicules in the Mg II $\mathrm{k}$ filtergram, at the instant of the images in Figure 1, where a view of these spicules is shown.

(A color version of this figure is available in the online journal.)

same loci of the spicule bushes from the $\mathrm{Mg}$ II raster. The Si IV $140 \mathrm{~nm}$ slit-jaw images show essentially the same.

In the bottom middle panel of Figure 4, one can see a TR image from the red wing of the Si IV $139.39 \mathrm{~nm}$ line where spicules and their disk counterparts are still visible. Many of such spicules are a continuation of the $\mathrm{Mg}$ II spicules, in particular when seen on disk. The most obvious example is the strongest bush in the raster, at $(x, y) \approx(50,25) \mathrm{Mm}$. In the $\mathrm{Mg}$ II images, the spicules are just below the reference line in the figure (cyan color), and in Si IV they extend more than $5 \mathrm{Mm}$ above the reference line. A closer look shows that the same is true for almost all spicule bushes, even though in some cases they are noticeably fainter in Si IV.

\section{DISCUSSION}

IRIS brings a comprehensive new look into the evolution and origin of spicules. The most clear result so far is that spicules undergo thermal evolution to at least TR temperatures. The fading of type II spicules seen by SOT is not observed in the IRIS filtergrams, and is in some cases associated with increased emission in the $\mathrm{Si}$ IV filtergrams. $\mathrm{Mg}$ II and $\mathrm{Si}$ IV spicules continue to rise for 4-8 Mm more, evolving for several more minutes after fading from Ca II. This strongly supports the idea that such spicules are undergoing heating and disappear from the Ca II $\mathrm{H}$ passband, as previously suggested (De Pontieu et al. 2009, 2011). Such evolution is also seen in the candidate spicule modeled by Martínez-Sykora et al. (2013), which disappears from Ca II $\mathrm{H}$ and continues in TR filtergrams. It is somewhat puzzling that the spicules in $\mathrm{Ca}$ II are so different from $\mathrm{Mg}$ II, as one would expect both elements to be predominantly doubly ionized at about the same temperatures $(\approx 15 \mathrm{kK}$; see Carlsson \& Leenaarts 2012). The persistence of $\mathrm{Mg}$ II while Ca II disappears could indicate time-dependent ionization effects or may stem from the much higher opacity of the $\mathrm{k}$ line ( $\mathrm{Mg}$ is about 18 times more abundant than $\mathrm{Ca}$, and the $\mathrm{k}$ line is about twice as strong as the $\mathrm{H}$ line). This warrants further investigation.

The Si IV spicules behave as one would expect with spicular heating to TR temperatures: they rise farther and are often brighter near the top. They extend above the Mg II spicules in nearly all cases, and undergo essentially the same time evolution (in some rare cases spicules rise and fade in $\mathrm{Mg}$ II while in Si IV they rise and $f a l l)$. The heating to TR temperatures is confirmed by the AIA $30.4 \mathrm{~nm}$ channel, which shows similar dynamics and evolution to the Si IV spicules, and is also seen in the spectral diagnostics. The presence of spicules in multiple filters underscores their multi-thermal nature, while the brighter tops in Si IV suggest heating.

Type II spicules show parabolic space-time diagrams in the IRIS and AIA filters, but should not be confused with type I spicules. The distinction between the two types made by De Pontieu et al. (2007) was that type II were faster, much shorter lived features that faded from $\mathrm{Ca}$ II $\mathrm{H}$ filtergrams. Our results confirm this. In addition, we now observe that this fading from $\mathrm{Ca}$ II $\mathrm{H}$ is associated with vigorous heating to higher temperatures. The fact that type II spicules continue evolving in the IRIS filtergrams does not change the fundamental differences between the two types of spicules. Some studies such as Zhang et al. (2012) claimed that all Ca II H spicules behave as type I (i.e., slow and do not fade from the filtergrams), but their results could not be reproduced by Paper I, who analyzed their data sets and instead found type II spicules dominant in quiet Sun and coronal holes. The two types should not be confused.

The group behavior of spicules is clearer in the IRIS observations. What is often perceived as one or two spicules in SOT turns out to be a much wider structure in IRIS (see Figure 1). With clearly defined multiple strands, these wide bunches of spicules have the same time evolution, and extend to areas with little or no emission in Ca II. Such group behavior plausibly contributes to the appearance of seemingly co-spatial spicules in lower-resolution observations (Pereira et al. 2013a). It is likely that only the cooler strands of such groups become visible in $\mathrm{Ca}$ II. The group behavior, multi-thermal nature, and apparent heating of these spicules indicate that these features are likely not driven in the same way as dynamic fibrils (Hansteen et al. 2006) even if the presence of parabolic paths is suggestive of the presence of a shock wave. Instead, the vigorous heating suggests some form of magnetic heating, perhaps as a result of magnetic reconnection (as suggested by De Pontieu et al. 2007).

Detailed spectra from IRIS rasters show that the disk counterparts of quiet Sun spicules are easily seen in the wings of chromospheric and TR lines. They rise in "bushes" from the magnetic network as previously reported (Langangen et al. 


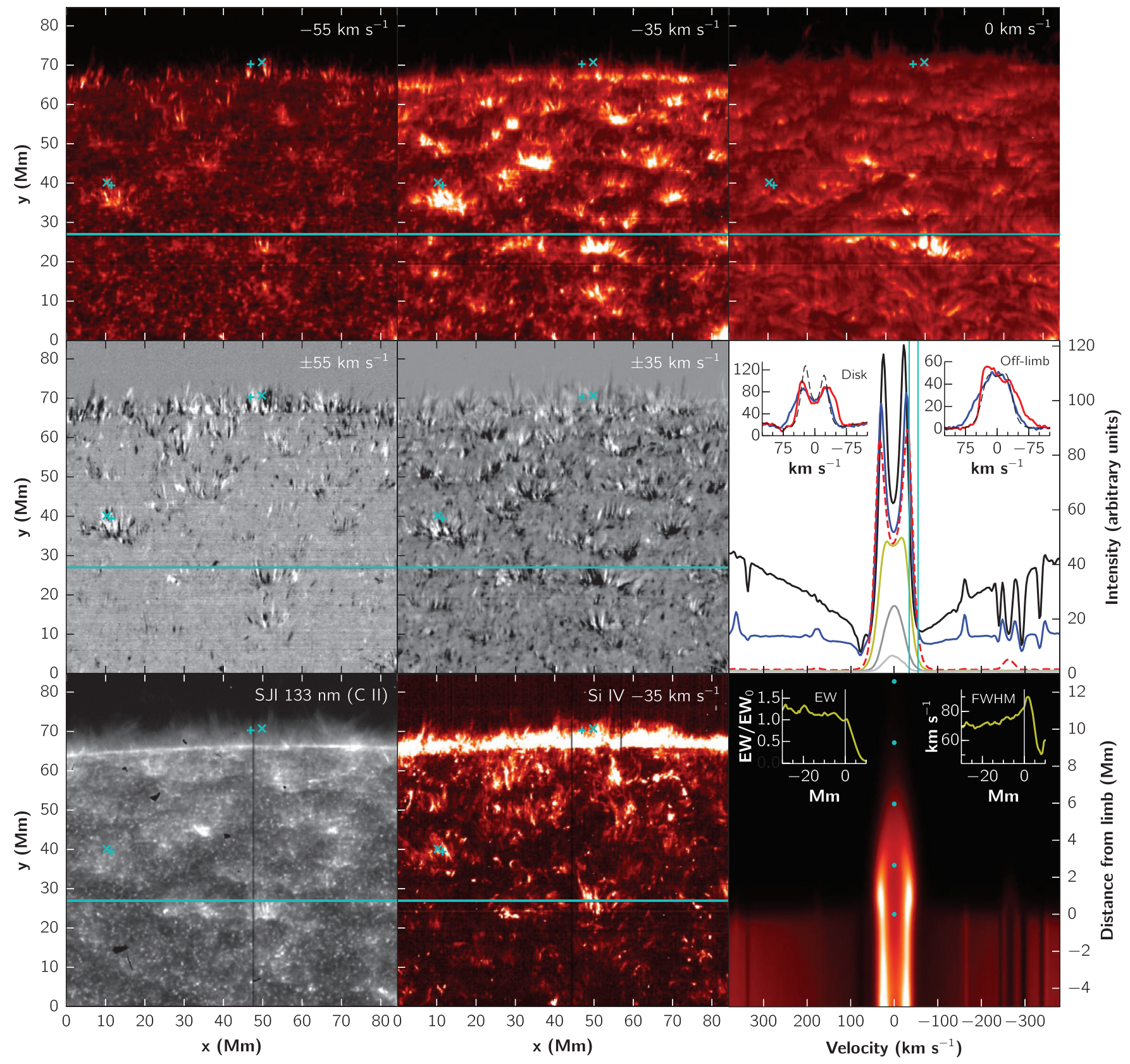

Figure 4. Synoptic view of a dense IRIS raster from 2013 October 9 at 13:10 UT. The size of the spatial window shown is approximately $84 \times 84 \mathrm{Mm}\left(116^{\prime \prime} \times 116^{\prime \prime}\right)$. Top three panels: raster intensity at fixed wavelengths or velocity shifts: $-55,-35$, and $0 \mathrm{~km} \mathrm{~s}^{-1}$ from the $\mathrm{Mg} \mathrm{II} \mathrm{k}$ line center. Middle left and center panels: Mg II $\mathrm{k}$ Dopplergrams at \pm 55 and $\pm 35 \mathrm{~km} \mathrm{~s}^{-1}$ (black and white mean positive and negative line-of-sight velocities, respectively). Middle right: Mg II k spectra and properties, with main part showing mean spectra at several distances from the limb (black: $16.9 \mathrm{Mm}$ below the limb, blue: at the limb, red dashed, yellow, gray and light gray: 2.7, 6.0, 9.3, and $12.6 \mathrm{Mm}$ above the limb, respectively. All but the first point are indicated by the cyan dots in the bottom right panel. The vertical cyan lines show the velocity positions at -55 and $-35 \mathrm{~km} \mathrm{~s}^{-1}$. The insets show spectra at the locations of the cyan symbols in the images, compared with the mean spectrum at the same distance from the limb (black dashed). There are two sets of points: on disk $((x, y) \approx(10,40) \mathrm{Mm})$ and off the limb $((x, y) \approx(50,70) \mathrm{Mm})$. The crosses indicate blue-shifted features and are plotted with a blue line, the plus signs show red-shifted features and are plotted in red. Lower left: $\mathrm{C}$ II $133 \mathrm{~nm}$ slit-jaw image taken around the middle of the raster. Lower center: raster intensity at $-35 \mathrm{~km} \mathrm{~s}^{-1}$ from the Si IV $139 \mathrm{~nm}$ line center. Lower right: spectrogram averaged along the direction parallel to the limb. The insets show the FHWM and equivalent width (EW) as a function of the distance from the limb (EW is normalized by $\mathrm{EW}_{0}$, the $\mathrm{EW}$ at the limb). All images shown in a linear scale, with different saturation thresholds.

(A color version of this figure is available in the online journal.)

2008; Rouppe van der Voort et al. 2009; Sekse et al. 2012; Yurchyshyn et al. 2013), and both the on-disk and off-limb features show the same spectral signatures as the RBEs/RREs (e.g., Sekse et al. 2013), providing more evidence that RBEs/RREs are the disk counterparts of spicules.

Spicules as depicted by IRIS are complex and multifaceted. A pattern of multi-thermal spicules with vigorous heating is emerging, and will place constrains on much needed theoretical modeling. The field is ripe for further studies.

IRIS is a NASA Small Explorer mission developed and operated by LMSAL with mission operations executed at NASA ARC and major contributions to downlink communications funded by the NSC (Norway). Hinode is a Japanese mission 
developed by ISAS/JAXA, with the NAOJ as domestic partner and NASA and STFC (UK) as international partners. It is operated in cooperation with ESA and NSC (Norway). This work was supported by the European Research Council grant No. 291058 and by NASA under contracts NNM07AA01C (Hinode), and NNG09FA40C (IRIS).

\section{REFERENCES}

Beckers, J. M. 1968, SoPh, 3, 367

Carlsson, M., \& Leenaarts, J. 2012, A\&A, 539, A39

De Pontieu, B., McIntosh, S., Hansteen, V. H., et al. 2007, PASJ, 59, 655

De Pontieu, B., McIntosh, S. W., Carlsson, M., et al. 2011, Sci, 331, 55

De Pontieu, B., McIntosh, S. W., Hansteen, V. H., \& Schrijver, C. J. 2009, ApJL, 701, L1

De Pontieu, B., Title, A. M., Lemen, J. R., et al. 2014, SoPh, 289, 2733

Dere, K. P., Bartoe, J.-D. F., \& Brueckner, G. E. 1989, SoPh, 123, 41

Hansteen, V. H., De Pontieu, B., Rouppe van der Voort, L., van Noort, M., \& Carlsson, M. 2006, ApJL, 647, L73

Kosugi, T., Matsuzaki, K., Sakao, T., et al. 2007, SoPh, 243, 3

Langangen, Ø., De Pontieu, B., Carlsson, M., et al. 2008, ApJL, 679, L167

Leenaarts, J., Pereira, T. M. D., Carlsson, M., Uitenbroek, H., \& De Pontieu, B. 2013a, ApJ, 772, 89

Leenaarts, J., Pereira, T. M. D., Carlsson, M., Uitenbroek, H., \& De Pontieu, B. 2013b, ApJ, 772, 90
Lemen, J. R., Title, A. M., Akin, D. J., et al. 2012, SoPh, 275, 17

Martínez-Sykora, J., De Pontieu, B., Leenaarts, J., et al. 2013, ApJ, 771, 66

McIntosh, S. W., \& De Pontieu, B. 2009, ApJ, 707, 524

McIntosh, S. W., Innes, D. E., de Pontieu, B., \& Leamon, R. J. 2010, A\&A, 510, L2

O’Shea, E., Banerjee, D., \& Doyle, J. G. 2005, A\&A, 436, L43

Pereira, T. M. D., De Pontieu, B., \& Carlsson, M. 2012, ApJ, 759, 18

Pereira, T. M. D., De Pontieu, B., \& Carlsson, M. 2013a, ApJ, 764, 69

Pereira, T. M. D., Leenaarts, J., De Pontieu, B., Carlsson, M., \& Uitenbroek, H 2013b, ApJ, 778, 143

Rouppe van der Voort, L., Leenaarts, J., de Pontieu, B., Carlsson, M., \& Vissers, G. 2009, ApJ, 705, 272

Sekse, D. H., Rouppe van der Voort, L., \& De Pontieu, B. 2012, ApJ, 752, 108

Sekse, D. H., Rouppe van der Voort, L., De Pontieu, B., \& Scullion, E. 2013, ApJ, 769,44

Sterling, A. C. 2000, SoPh, 196, 79

Suematsu, Y., Tsuneta, S., Ichimoto, K., et al. 2008, SoPh, 249, 197

Tian, H., DeLuca, E., Cranmer, S. R., et al. 2014, Sci, submitted

Tian, H., McIntosh, S. W., Habbal, S. R., \& He, J. 2011, ApJ, 736, 130

Tsiropoula, G., Tziotziou, K., Kontogiannis, I., et al. 2012, SSRv, 169, 181

Tsuneta, S., Ichimoto, K., Katsukawa, Y., et al. 2008, SoPh, 249, 167

Wilhelm, K. 2000, A\&A, 360, 351

Yurchyshyn, V., Abramenko, V., \& Goode, P. 2013, ApJ, 767, 17

Zachariadis, T. G., \& Gontikakis, C. 2002, in SOLMAG 2002, Proceedings of the Magnetic Coupling of the Solar Atmosphere Euroconference, ed. H. Sawaya-Lacoste (ESA SP-505, Noordwijk: ESA), 619

Zhang, Y. Z., Shibata, K., Wang, J. X., et al. 2012, ApJ, 750, 16 